\title{
Framework for propagating stress control message using heartbeat based IoT remote monitoring analytics
}

\author{
Eisha Akanksha \\ Department of Electronics and Communication, CMR Institute of Technology, India
}

\begin{tabular}{|c|c|}
\hline Article Info & ABSTRACT \\
\hline & \multirow{10}{*}{$\begin{array}{l}\text { Abnormal level of stress is the root indicator factor to have significant impact } \\
\text { over the health of heart and there is a close relationship between the stress } \\
\text { levels with heart rate. Review of the existing literature showcase that there } \\
\text { has been various work that has been carried out towards investigation of } \\
\text { considering heart rate with an internet-of-things (IoT) system. Apart from } \\
\text { this, existing system doesnt offer any instantaneous solution where certain } \\
\text { intimation is offered in real-time to the user with wearables as a solution to } \\
\text { control the stress condition. Therefore, the current paper introduces a novel } \\
\text { framework where the sampled heart rates of the patients are captured by IoT } \\
\text { deivices. The aggregated data are further forwarded to the cloud analytic } \\
\text { system that uses correlation to extract the appropriate message. The system } \\
\text { after being applied with teh machine learning approach could further extract } \\
\text { the elite outcome followed by forwarding the contextual data to teh user. } \\
\text { Using an analytical modelliig, the proposed system shows that it offers better } \\
\text { accuracy and reduced processing time when compared with other machine } \\
\text { learning approach and thereby it proves to be cost effective solution in IoT } \\
\text { system over medical case study. }\end{array}$} \\
\hline Received Jul 26, 2019 & \\
\hline Revised Feb 29, 2020 & \\
\hline Accepted Mar 18, 2020 & \\
\hline Keywords: & \\
\hline Heartbeat & \\
\hline Internet-of-things & \\
\hline Machine learning & \\
\hline Medical IoT & \\
\hline Remote monitoring & \\
\hline
\end{tabular}

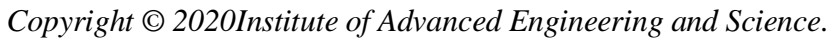
All rights reserved.

\section{Corresponding Author:}

Eisha Akanksha,

Department of Electronics and Communication,

CMR Institute of Technology,

Bengaluru, India.

Email: eisha.a@cmrit.ac.in

\section{INTRODUCTION}

The internet-of-things (IoT) is the next upcoming technology that enables inter-connectivity of multiple number of devices (or smart devices) where majority of the devices are basically sensors [1, 2]. One of the significant capabilities of the IoT is to perform sensing of the information remotely with an aid of using multiple forms of infrastructure of the network [3]. IoT thereby allows integration of various devices that are directly connected to the physical world and it also claims of offer better accuracy as well as cost effective measures $[4,5]$. Once various embedded systems are connected with the IoT, it transforms to a suitable IoT ecosystem. At present, healthcare system is undergoing a tremendous revolution with the proliferation of the usage of the IoT architecture offering maximum device-to-device connection. The important aspect of the contribution of IoT in healthcare sector is to ensure acquisition of real-time health data, forward it to the destination node, followed by the subjective analytical operation. The usage of IoT application could possibly use cloud environment if a large stream of data is required to be transmitted, stored and analyzed [6]. Basically, the ufosage of IoT in the medical sector can perform data acquisition from different users as well as patient in order to resist further spreading of the disease. Inclusion of medical IoT introduces various hardware-based devices that can perform improved quality of clinical diagnosis. The primary intention of usage of IoT is to reduce the mortality rate by i) real-time remote monitoring system of the patient, ii) offering comprehensive analysis of any form of critical diseases, and iii) performing predictive analysis of the occurances of any life-threatening medical condition. At present, there are various 
discussion of application idea concerning with the IoT system viz. i) body wearable, ii) remote health monitoring, iii) equipments to maintain vital statistics, iv) patient-oriented medicine, v) monitoring of medical assets, and vi) advanced analytics of the medical data [7].

At present, there has been various research work being carried out towards the discussion of the various strategies adopted for improving medical sector with inclusion of IoT [8-10]. However, there are various practical problems associated with the usage of the medical IoT. The first problem of medical IoT is the usage of sensors and body wearable. Irrespective of evolution of various forms of sensors in micro sizes, it cannot be used for all the medical condition. Another biggest problem is that different number of sensor acquires different data form and hence body wearable could offer discomfort and burden over the patient just for acquiring different type of bio-signals if they are required. Another problem associated with the medical IoT is the mechanism of transmission. Once the IoT device captures the information from the sensors or actuators, it usually forwards the information without any forms of processing on it. This causes a problem when there is a need of performing processing on the acquired data. The third problem associated with the medical IoT is the application design of the analytical program. Development of sophisticated analytics calls for comprehensive consideration of intention of the application design. At present, majority of the current application calls for acquiring the data followed by storing and managing the data in server end. However, certain system uses such analyzed data for the purpose of subjecting the data to the analysis where certain information is derived and passed on to the service provider in order to act upon. However, such application normally does no communicate with the user and such information is not even user friendly. Therefore, this paper presents a discussion of the novel solution that perform forwarding of the contextual message to the patient for the purpose of resisting the possible situation of stress. Section 1 discusses about the existing literatures where different techniques are discussed for detection schemes used in power transmission lines followed by discussion of research problems and proposed solution. Section 2 discusses about algorithm implementation followed by discussion of result analysis in Section 3. Finally, the conclusive remarks are provided in Section 4.

This section discusses about the existing approaches where researchers have used heartbeat data combined with IoT for facilitating healthcare sector with better advantage of clinical guidance. The recent work of Majumder et al. [11] have developed a predictive system for assiting forecasting of the warning for heart attack using wearable device over IoT. Zhang et al. [12] have developed a remotely tracking of patient health using IoT integrated with sensor network. Discussion of wearable device towards the usage in healthcare system in carried out by Al-Eidan et al. [13]. Similar form of discussion has been carried out by Meharouech et al. [14]. Mahmoud et al. [15] have performed an investigation towards cloud-of-things architecture associated with the healthcare system. The authors have mainly emphasized over energy efficiency factor in this presented system. Espinilla et al. [16] has discussed about the decision making framework that is capable of extracting knowledge over the bio-signals captured from the wearable applications in IoT. Study towards integration of wearbale device and IoT was also carried out by Monton et al. [17] using hardware-based approach in order to offer reduction in delay.

Another framework developed by Pasha et al. [18] emphasizing over the interoperability in the system design of healthcare. Adoption of fog computing integrated with an IoT towards developing a better form of healthcare system was carried out y Paul et al. [19] for increasing the efficiency of the system associated with the healthcare monitoring system. Wu et al. [20] has presented model for retaining energy efficiency of the system developed with wearable IoT system. The authors have claimed of their enhanced applicability towards mobile application during critical situation. Guan et al. [21] have presented another system design which assists the elderly patient remotely where a home gateway system is positioned to trace the critical limits of the heartbeats signals. A specific prototype of healthcare monitoring has been developed by Desai and Toravi [22] using hardware-based system. Ali et al. [23] have developed a system that conjoins sensor network with mobile device for monitoring patient health condition on the basis of its pulse. Similar form of study has been also carried out by Poorvi et al. [24] and Kumar et al. [25]. Sathishkumar et al. [26] have developed a prototype using IoT integrated with heartbeat sensor in order to resist uneven situation that could make the road safety vulnerable. The study carried out by Irawan and Juhana [27] using nearly the similar approach for tracking the heart rate of the patient using signal processing approach. Discussion of the healthcare-based application has been carried out by Rodriques [28] as well as there are also researchers that has focused on the security aspect of similar form of integrated IoT sensor healthcare application as that seen in work of Yeh [29]. The work carried out by Fouhad [30] has developed nearly similar prototype application focusing on the supportability of the telemedicine. Therefore, there are various studies being carried out where heartbeat sensors were used for developing an IoT framework for healthcare sector. Hamza et al. [31] have introduced a technique for smart car parking based on cloud method containing various types of sensor. The PIRs has been employed to detect the object motion.Bhagchandani and Augustine [32] have discussed a holistic solution by using the IOT method including data analytics.

Int J Elec \& Comp Eng, Vol. 10, No. 5, October 2020 : 4615 - 4622 
The IoT allows real-time capturing and calculation of the medical data from smart sensors installed in wearable devices. The work done by Wani and Revathi [33] the expansion of ransomware attacks for ransomwares threatening IoT. An identification tool for IoTransomware attack is demonstareted that is designed after reviewed of ransomware for the IoT. The presented technique shows the present IoT traffic through SDN gateway. It utilizes policies borders in SDN organize for sensing of ransomware in the IoT.

The potential research problems associated with the existing system are as follows:

- Existing approaches of using wearable devices and IoT is restricted only by receiving the heartbeat signal while not processing them for better clinical / motivational inference.

- At present, the studies do not discrete use machine learning techniques to arrive at user friendly suggestion in faster track meant exclusively for user.

- Assessing the reliability of the study using accuracy parameter and faster receiving of the signals in presence of a computational modeling is absent.

- Existing approaches mainly uses prototyping scheme where only narrowed area of implementation can be assessed.

Therefore, the problem statement of the proposed study can be stated as "Developing a framework that can facilitate user-friendly inference to countermeasure against their stress level with cost effective modeling is a complex and challenging task". The next section highlights the solution for this.

The prime aim of the proposed system is to offer a novel design of a framework that offers contextual services considering the case study of healthcare sector integrated with IoT system. The implementation of the proposed system is carried out using analytical research methodology where the prime concern is to offer accurate forwarding of the contextual messages to the user under variable stress condition in order to beat the critical. The schematic diagram of the proposed system is shown in Figure 1.

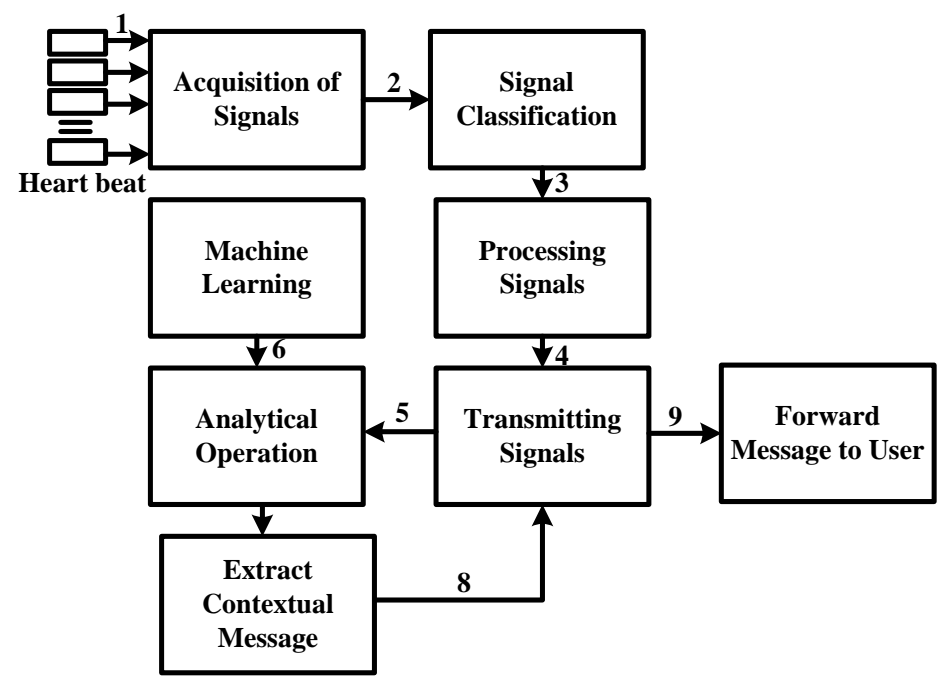

Figure 1. Proposed schema of implemented methodology

Figure 1 highlights the adopted schema of implementation. The core modules of the proposed system are acquisition of signals, processing of signals, transmitting the signals, followed by analysis of the signal. The proposed system considers the input as heartbeat of the user as the core indicators of stress. It is known that different levels of heartbeat consider different levels of stress. An IoT environment is constructed that connects the user's wearble device, extracts all the continuous data of heartbeat, followed by further processing the data. The proposed system than perform matching with the data with the clinical reference in order to ensure if the heartbeat signals obtains are critical or under critical. Only the critical data are extracted and indexed for shapping it in the form of query signals. The newly indexed query signals are then transmitted to the cloud-application where the analytics are running. The on-cloud analytics retains the list of all the specific messages in the form of clinical stress hierarchy. Correlation-based analysis is performed in order to match the filtered query signals with that maintained in the cloud. After the outcome of specific message is obtained, a machine learning approach is offered to perform training towards the obtained correlated data. This training process finally offers accurate contextual message that is forwarded to the user via network over the communication device of the user. 


\section{SYSTEM DESIGN}

The complete design of the proposed system is constructed using divide and conquers approach that mainly emphasize over obtaining accuracy of the vital stat e.g. heart beat of the user. Different individual modules of design have been constructed in order to develop the proposed model. This section discusses about assumption-dependencies, implementation strategy, and execution flow of proposed system as follows:

\subsection{Assumptions and dependencies}

The first assumption of the proposed study is that there is a synchronized set up of the wearable body sensors with the various IoT devices forming a smart healthcare analytic system. There are also good possibilities of using different forms of wearable sensors connected with each other and form a body area network. The second assumption of the proposed system is that heartbeat data is continuously monitored by the sensors; however, the filtration of the acquired signal corresponding to the severity of the stress condition is carried out by the sensor and not by the IoT gateway that receives the signal. The third assumption of the study is that complete data is forwarded through a secured and error free communication channel over predefined IoT environment. Similarly, there are various dependencies of the proposed system e.g. i) an error-free communication channel is required for performing transmission of the acquired heartbeat signal to the destination node, ii) There is also a dependency of elastic storage capacity in order to retain all the trained information obtained from the training phase. The trained data will be used for performing validation of accuracy as performance measures of proposed forecasted contextual message. iii) The final dependency of the proposed system is that there should be a hierarchical list of clinical/motivational suggested message that are specific to actual medical context of the captured heartbeat of the user.

\subsection{Implementation strategy}

The implementation of the proposed system is carried out by sequential module design which is meant for performing following steps of operation with defined strategies:

- Acquisition of signals

The proposed study considers that a user is wearing a body wearable sensor that can measure the true heart beats shown in Figure 2. The study considers that there are three categories of heartbeat viz. i) category-1: High beat rate ( $>100$ beats per minute), ii) category-2: Medium beat rate (100-60 beats per minute), and iii) category-3: ( $<60$ beats per minute). The heart beat is a continuous signal which the sensor captures followed by segregating the signals on the basis of the categories and then it is further subjected to processing in the next step.

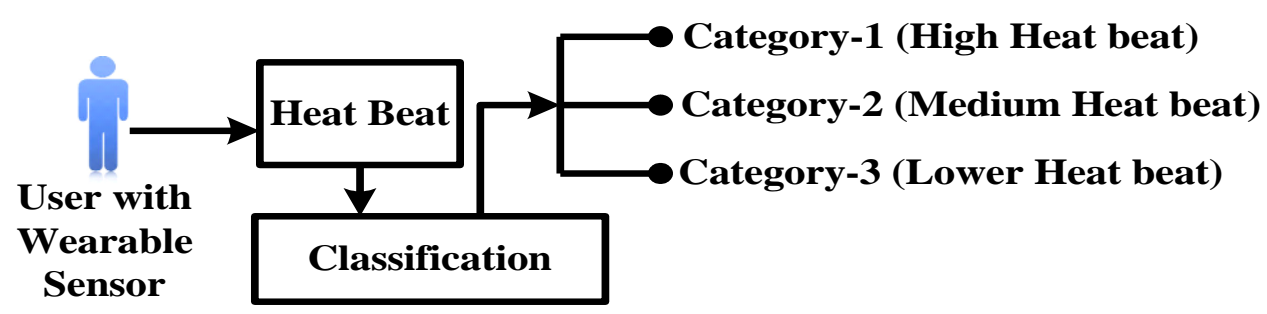

Figure 2. Acquisition of signal

- Processing the signals

The sensors are also considered to posses a capability to perform processing of the heart beat signals that corresponds to unique levels of stress of the user. For this purpose, the system considers that fact of possible situation of stress level is associated with either tachycardia or bradycardia, which is both considered as lethal conditional. Such condition will require an immediate medical attention. All the signals of heartbeats will be forwarded to different carriers of communication channel between wearable sensor and nearest IoT device (or IoT gateway system). It is to be understood that IoT gateway also acquire signals from other mobile users and will be responsible for further processing it. By processing, it will mean that a module is constructed that considers the category- 1 and category- 3 signals and time-stamps it. This signal is then further indexed in the form of two specific query systems. The first query is formulated for category-1 signal while the second query is formulated for cateogory-3 signal. Therefore, the processing of this signal is carried out for only critical data i.e. for category- 1 and category- 3 while leaving the non-critical signal i.e. category-2. Figure 3 offers pictorial representation of this operation. 
- Transmitting the signals

The transmission of the filtered signal from the prior stage is carried out in this module. The filtered signal mainly consists of indexed signal of category-1 and category-3 where the indexing will mean embedding the respective heartbeat with time stamp and user identity. The fused data is now forwarded to the IoT gateway node followed by forwarding the same to the access point and edge server. However, while doing so, there is quite a possibility that any of this networking device (e.g. gateway node, access point, edge server) is found to be currently processing a job. In such condition, an adaptive queue system is considered where the incoming task is reposited for immediate processing. Another interesting part of this transmission module is that it can also support prioritizing the any specific incoming signals using two attributes. The first attribute for prioritizing the incoming signal will be based on certain cut-off level of heart beat. An additional much-critical cut-off level can be given to further develop an additional queue for such incoming signals and they will be first processed followed by prior queue. The second attribute for prioritizing will be wait-time of queue (such situation can occur due to network problems or high density traffic). If a signal packet is found to wait for more than a specific duration of time, it will be soon pushed to priority queue. The end result of this module is that the fused data are successfully transmitted to the cloud application that runs analytics for further analyzing the fused signals. Figure 4 represents the pictorial way of this transmission process.

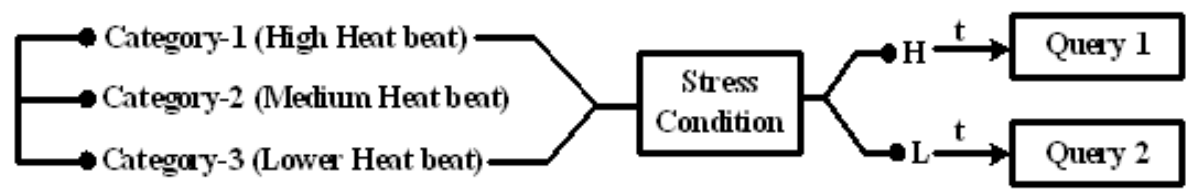

Indexed Bio-Signals

Figure 3. Processing the signals

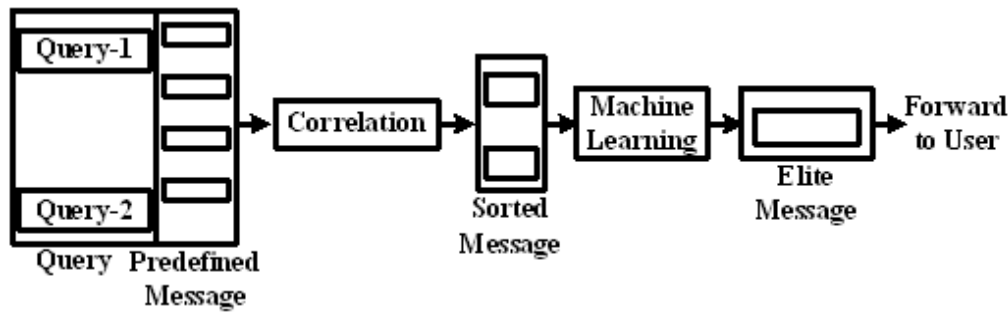

Figure 4. Transmission of signals

- Analysis of signals

This is the last phase of implementation that emphasize on yielding a definitive motivational / clinical message (or suggestion) to the respective users on the basis of obtained signals shown in Figure 5. The study consider a present of distributed motivational / clinical message maintained over the storage units with a preamble matching with specific condition of the heart beat. The proposed system applies a correlation of the obtained signal with the correlated value of the messages and only the highly correlated value is shortlisted. However, in order to prevent any form of false positive, the proposed sysem applies machine learning algorithm that performs training operation in order to validate the outcome resulted from correlation and finally obtain the elite message to be forwarded to user.

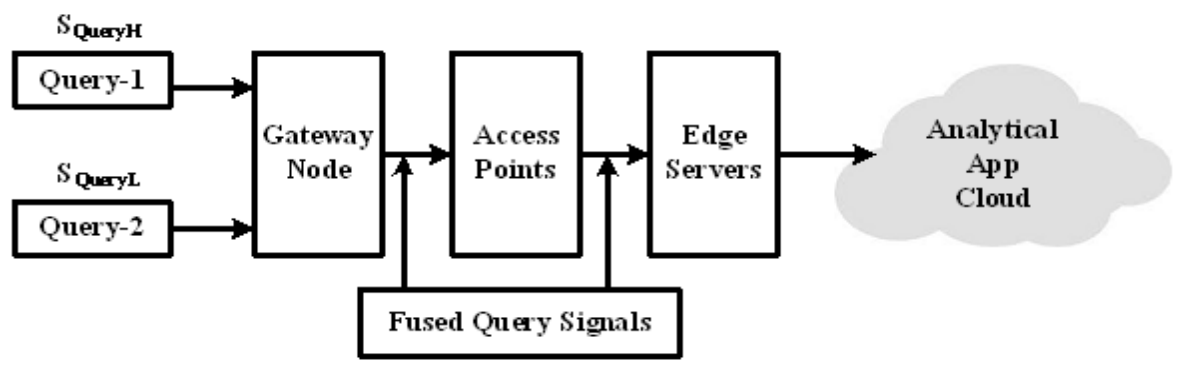

Figure 5. Analysis of signals 


\subsection{Execution flow}

The proposed system constructs an algorithm that is responsible for transmitting the contextual message over IoT environment on the basis of the heart beat data. The algorithm takes the input of $d$ (sensory data) and $H / M / L$ (high/medium/low heartbeat) which after processing will yield an outcome of $\mu$ (contextual message). The steps of algorithmic execution are as follows:

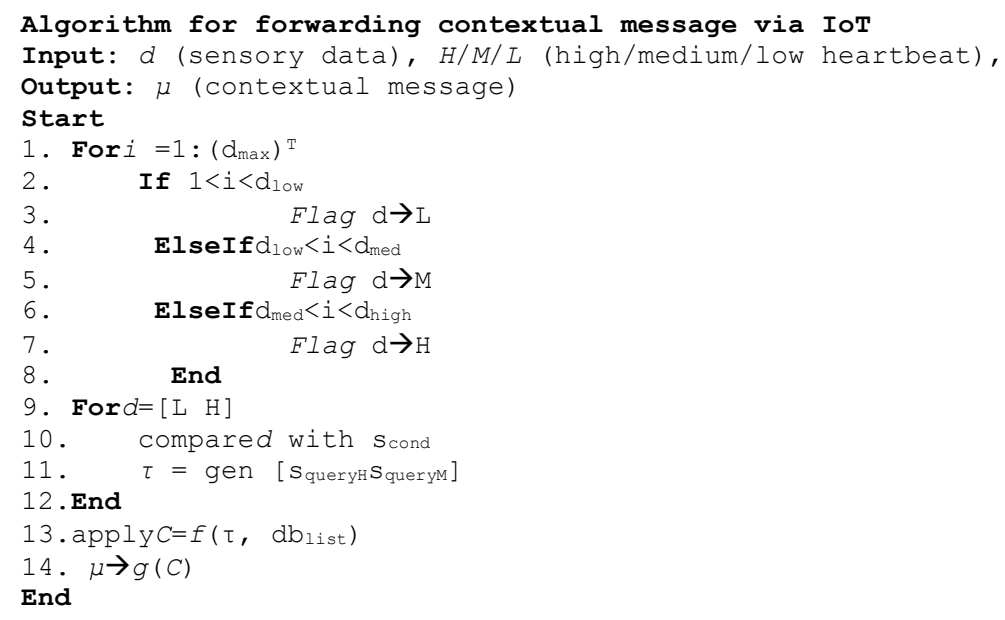

The discussion of the execution flow of the proposed algorithm is as follows: Assuming that a body wearable sensor device has captured heart-beat data $\mathrm{d}$ using a sample period of $\mathrm{T}$ and the sampled data is forwarded to the IoT gateway node in the proximity. Consider that $\mathrm{d}_{\max }$ is the maximum size of the sampled heat-beat data; the algorithm evaluates the type of the heart beat data in terms of cut-off. If the heart-beat is found to be with low cut-off $d_{\text {low }}$ (Line-2) than the filtered data $d$ is flagged as $L$ (Line-3). Similarly, the heart-beat data $d$ is compared with medium $\left(\mathrm{d}_{\text {med }}\right)$ and high $\left(\mathrm{d}_{\text {high }}\right)$ cut-off to flag $M$ and $H$ respectively (Line-5 and Line-6). For an effective analysis, the algorithm ignores $\mathrm{M}$ signal but considers $\mathrm{L}$ and $\mathrm{H}$ signal. A temporary buffer space is created that retains only $\mathrm{L}$ and $\mathrm{H}$ (Line-9). The algorithm also constructs a clinical condition $\mathrm{s}_{\text {cond }}$ to define the degree of criticality of the $\mathrm{L}$ and $\mathrm{H}$ type of signal of heartbeat and this condition is a referential point of stress factor too. A consideration of tachycardia ( $<60$ beats per minute) and bradycardia (more than 100 beats per minute) is also considered while forming the conditional function $\mathrm{s}_{\text {cond }}$ (Line-10) in order to further confirm the severity of stress as both the conditions are considered to be dangerous. The system then formulates a function to generate the normalized heart rate corresponding to $\mathrm{H}$ and $\mathrm{M}$ and is represented as $\mathrm{s}_{\text {queryH }}$ and $\mathrm{s}_{\text {querym }}$ respectively (Line-11). The query matrix $\tau$ retained

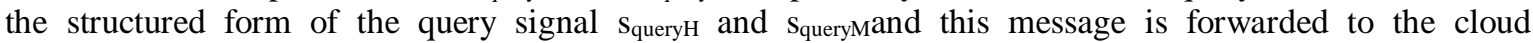
aanalytical application via gateway node, access point, and edge server. This process of query generation is repeated for all the value of sampled sensory data $\mathrm{M}$ and $\mathrm{H}$ (Line-9 to Line-12). After the matrix $\tau$ is obtained in the cloud analytical application it matches with the hierarchical list of pre-defined messages $\mathrm{db}_{\text {list }}$. It should be noted that there are different forms of prediefined messages unique made for each categories of queries. The algorithm than applies correlation function $f(\mathrm{x})$ over received data $\tau$ with list of pre-defined messages dblist (Line-13). As correlation results in sorted value of higher correlated data as an output; therefore there are chances that such outcome could also have outliers. Apart from this, as there are various types of disease condition that could also have similar pattern of heart beat. Therefore, forwarding same contextual message could be proved as false positive. This problem is solved using machine learning approach, where training is carried out considering specific charecteristics of user's data along with the generated heart beat data in order to conclude the elite outcome. The elite outcome will mean that the algorithm successfully concluded about one message from the list of message that is highly suitable for the user in its current health condition or called as real-time contextual data. The proposed system applies a machine learning function $g(\mathrm{x})$ considering the contextual data $\mathrm{C}$ as the input argument in order to generate an elite outcome $\mu$ (Line-14). Therefore, the proposed execution of the algorithm is highly a progressive step with an inclusion of only one iterative step during training process which also maintains a higher degree of cost effectiveness while processing the heartbeat signal for the purpose of forwarding the correct contextual messages to the user via any communicating devices.

\section{RESULT ANALYSIS}

The scripting of the proposed system has been carried out using MATLAB considering the heart rate dataset [34]. As the proposed system deals with offering the precise contextual message for user, therefore, 
it is essential to validate if the correct contextual data has been received and if it has been received on time. Therefore, the core performance parameter considered are accuracy of choosing the right contextual message and processing time that defines the duration spend from acquiring the heart beat data to receiving of contextual message to the user. The proposed system also performs comparative analysis with the existing machine learning approach to find the best approach.

The study outcome clearly shows that deep neural network offers better advantage with respect to the accuracy shown in Figure 6 and processing time shown in Figure 7. The core reason for the higher accuracy is its capability to recognize the error even if the error is outside of the tolerance level. This feature is not present in other forms of machine learning approach. The prime reason behind this is increased training time involved in neural network and support vector machine (SVM) for matching the best result with the correlated data maintained over the list. Therefore, it can be said that proposed system offers cost effective solution where the contextual data is accurately generated as well as incurs less time to forward the correct contextual message to the user and hence it offers practical advantage over critical clinical requirement.

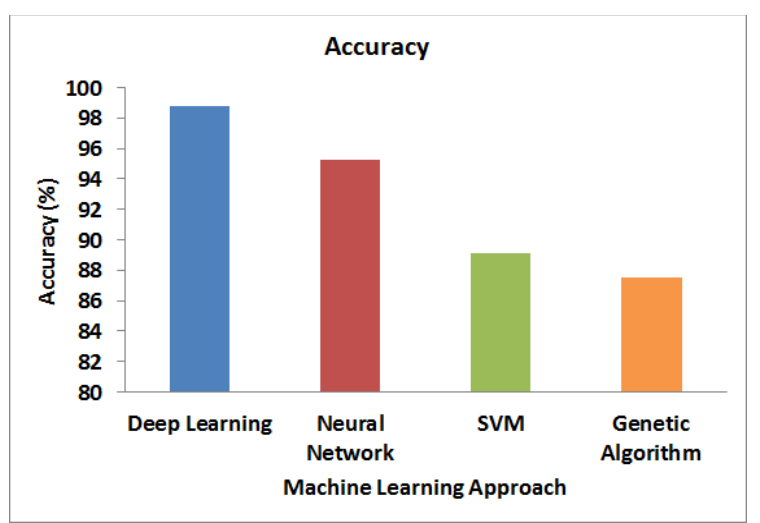

Figure 6. Comparative analysis of accuracy

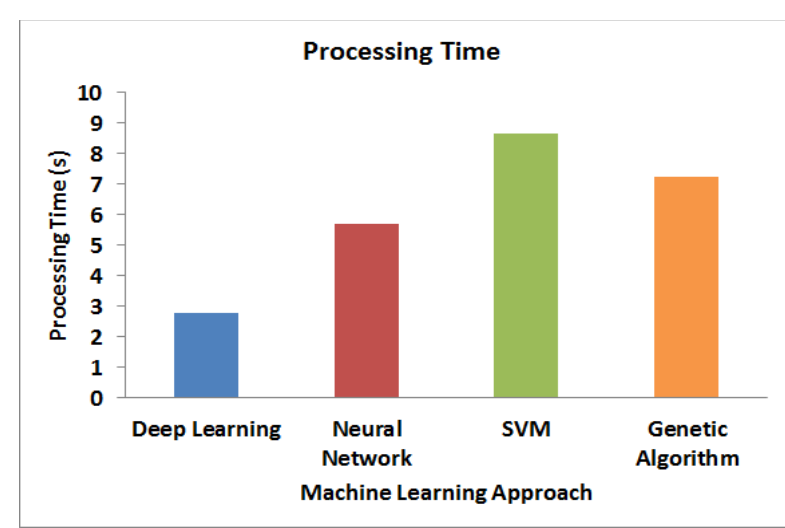

Figure 7. Comparative analysis of processing time

\section{CONCLUSION}

Acquisition of the bio-signals can be suitably used for controlling stress factor with an aid of IoT. However, processing the bio-signals like heartbeats is an important task that requires a cost effective method for transforming the signals over the presence of analytics. Therefore, the proposed system introduces a mechanism that extracts the heartbeat signal, processes it, and transmits it to the edge server via IoT gateway system. The proposed system uses correlation-based approach in order to extract the definitive motivational/clinical suggestion that are meant to be directly forward to the user in order to instantly control the level of stress. Further machine learning approach is used for further extracting the accurate contextual message. The simulated outcome of the study proves that proposed system offer good accuracy and reduced processing time to do the entire operation.

\section{REFERENCES}

[1] Staudemeyer, Ralf C., H. C. Pöhls and B. W. Watson, "Security and privacy for the internet of things communication in the smartcity," Designing, Developing, and Facilitating Smart Cities, pp. 109-137, 2017.

[2] Pagel, M. and D. Carlson, "Ambient control: A mobile framework for dynamically remixing the internet of things," IEEE 16th International Symposium on A World of Wireless, Mobile and Multimedia Networks, pp. 1-9, 2015.

[3] Chen, Hao, Xueqin Jia and Heng Li, "A brief introduction to IoT gateway," IET International Conference on Communication Technology and Application, pp. 610-613, 2011.

[4] Aazam, Mohammad and Eui-Nam Huh, "Fog computing: The cloud-iotVioe middleware paradigm," IEEE Potentials, vol. 35, no. 3, pp. 40-44, 2016.

[5] Agrawal, Sarita and Manik Lal Das, "Internet of Things-A paradigm shift of future Internet applications," 2011 Nirma University International Conference on Engineering, pp. 1-7, 2011.

[6] $\mathrm{He}, \mathrm{Wu}$, Gongjun Yan and $\mathrm{Li} \mathrm{Da} \mathrm{Xu}$, "Developing vehicular data cloud services in the IoT environment," IEEE Transactions on Industrial Informatics, vol. 10, no. 2, pp. 1587-1595, 2014.

[7] Chiuchisan, Iuliana, Hariton-Nicolae Costin and O. Geman, "Adopting the internet of things technologies in health care systems," International Conference and Exposition on Electrical and Power Engineering, pp. 532-535, 2014.

[8] Turcu, Cristina Elena and Cornel Octavian Turcu, "Internet of things as key enabler for sustainable healthcare delivery," Procedia-Social and Behavioral Sciences, vol. 73, pp. 251-256, 2013. 
[9] Ray, Partha Pratim, "Understanding the role of internet of things towards smart e-healthcare services," Biomedical Research, vol. 28, no. 24, 2017.

[10] Polonetsky, Jules and Stacey Gray, "The internet of things as a tool for inclusion and equality," Fed. Comm. pp. 103-118, 2017.

[11] Majumder, A. K. M., Yosuf Amr ElSaadany, R. Young, and D. R. Ucci, "An energy efficient wearable smart iot system to predict cardiac arrest," Advances in Human-Computer Interaction, 2019.

[12] Zhang, Weiping, M. Kumar, J. Yu, and J. Yang, "Medical long-distance monitoring system based on internet of things," EURASIP Journal on Wireless Communications and Networking, vol. 2018, no. 1, pp. 1-8, 2018.

[13] Al-Eidan, Rasha M., Hend Al-Khalifa and Abdul Malik Al-Salman, "A review of wrist-worn wearable: Sensors, models, and challenges," Journal of Sensors, vol. 2018, pp. 1-20, 2018.

[14] Meharouech, Amira, Jocelyne Elias and Ahmed Mehaoua, "Moving towards body-to-body sensor networks for ubiquitous applications: A survey," Journal of Sensor and Actuator Networks, vol. 8, no. 2, pp. 27, 2019.

[15] Mahmoud, Mukhtar M. E, et al., "Enabling technologies on cloud of things for smart healthcare," IEEE Access, vol. 6, pp. 31950-31967, 2018.

[16] Espinilla, Macarena, Javier Medina, Ángel-Luis García-Fernández, Sixto Campaña, and Jorge Londoño, "Fuzzy intelligent system for patients with preeclampsia in wearable devices," Mobile Information Systems, 2017

[17] Bayo-Monton, et al, "Wearable sensors integrated with internet of things for advancing eHealth care," Sensors, vol. 18, no. 6, pp. 1-18, 2018.

[18] Pasha, Maruf, and Syed Muhammad Waqas Shah, "Framework for E-Health systems in IoT-based environments," Wireless Communications and Mobile Computing, 2018.

[19] Paul, Anand, Hameed Pinjari, Won-Hwa Hong, Hyun Cheol Seo, and Seungmin Rho, "Fog computing-based IoT for health monitoring system," Journal of Sensors, vol. 2018, pp. 1-7, 2018.

[20] Wu, Fan, Jean-Michel Redouté, and Mehmet Rasit Yuce, "We-safe: A self-powered wearable IoT sensor network for safety applications based on LoRa," IEEE Access, vol. 6, pp. 40846-40853, 2018.

[21] Guan, Kai, Minggang Shao, and Shuicai Wu, "A remote health monitoring system for the elderly based on smart home gateway," Journal of healthcare engineering, vol. 2017, 2017.

[22] Desai, M. R., and Sushma Toravi, "A smart sensor interface for smart homes and heart beat monitoring using WSN in IoT environment," International Conference on Current Trends in Computer, Electrical, Electronics and Communication, pp. 74-77, 2017.

[23] Ali, N.S., et al., "Real-Time heart pulse monitoring technique using wireless sensor network and mobile application," International Journal of Electrical and Computer Engineering (IJECE), vol. 8, no. 6, 2018.

[24] Jayanth, S., M. B. Poorvi, R. Shreyas, B. Padmaja and M. P. Sunil, "Wearable device to measure heart beat using IoT," International Conference on Inventive Systems and Control, pp. 1-5, 2017.

[25] Kumar, G. Vijay, A. Bharadwaja and N. Nikhil Sai, "Temperature and heart beat monitoring system using IoT," International Conference on Trends in Electronics and Informatics, pp. 692-695, 2017.

[26] Pughazendi, N., R. Sathishkumar, S. Balaji, S. Sathyavenkateshwaren, S. Subash Chander, and V. Surendar, "Heart attack and alcohol detection sensor monitoring in smart transportation system using Internet of Things," International Conference on Energy, Communication, Data Analytics and Soft Computing, pp. 881-888, 2017.

[27] Irawan, Hendry Cahya and Tutun Juhana, "Heart rate monitoring using IoT wearable for ambulatory patient," 11th International Conference on Telecommunication Systems Services and Applications, pp. 1-4, 2017.

[28] Rodrigues, Joel JPC, et al., "Enabling technologies for the internet of health things," IEEE Access, vol. 6, pp. 13129-13141, 2018

[29] Yeh, Kuo-Hui, "A secure IoT-based healthcare system with body sensor networks," IEEE Access, vol. 4, pp. 10288-10299, 2016.

[30] Fouad, Hafez, and Hesham Farouk, "Heart rate sensor node analysis for designing internet of things telemedicine embedded system," Cogent Engineering, vol. 4, no. 1, pp. 1-12, 2017.

[31] Hamzah, Abdulwahhab Essa Hamzah, "Design and implement WSN/IOT smart parking management system using microcontroller," PhD Thesis, Universiti Tun Hussein Onn Malaysia, 2018.

[32] Bhagchandani, Khushboo and D. Peter Augustine, "IoT based heart monitoring and alerting system with cloud computing and managing the traffic for an ambulance in India," International Journal of Electrical and Computer Engineering (IJECE), vol. 9, no. 6, pp. 5068-5074, 2019.

[33] Wani, Azka, and S. Revathi, "Ransomware protection in loT using software defined networking," International Journal of Electrical and Computer Engineering (IJECE), vol. 10, no. 3, pp. 3166-3175, 2020.

[34] "BIDMC PPG and Respiration Dataset," [online], Avaible: https://physionet.org/pn6/bidmc/, [Retrieved on 27-06-2019].

\section{BIOGRAPHY OF AUTHOR}

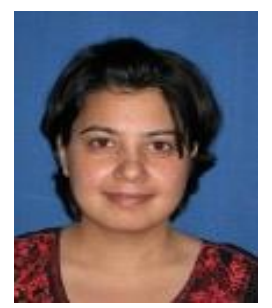

Eisha Akanksha, she is an associate professor, Department of Electronics \& Communication, CMRIT, Bengaluru, India. Her areas of interest are wireless communications, Light Fidelity, Internet of Things and Cloud Computing. She has around 6 years of experience in teaching field. She has worked 2.4 Years of Industry Experience- Worked as software engineer in Sasken Technologies Bangalore, India. 